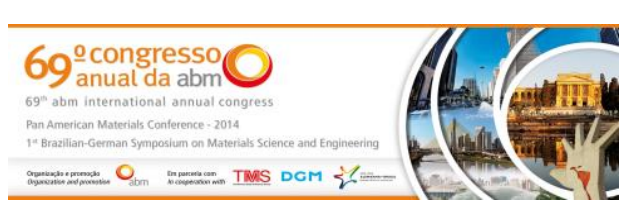

Tema: Diagramas de fases e transformações de fases

\title{
CARACTERIZAÇÃO MICROESTRUTURAL E MECÂNICA PARA QUANTIFICAÇÃO DA MARTENSITA INDUZIDA POR DEFORMAÇÃO DE UM AÇO INOXIDÁVEL AUSTENÍTICO 304L*
}

Dielle Cristine Toledo Costa ${ }^{1}$ Gláucio Soares da Fonseca²

\section{Resumo}

Os aços austeníticos 304L quando processados tem suas propriedades mecânicas afetadas pelas transformações martensíticas induzidas pela deformação plástica (efeito TRIP). O objetivo deste trabalho foi avaliar esse comportamento em corpos de prova que sofreram deformações gradativas por meio de ensaios de tração uniaxial a partir de diferentes técnicas de análises microestruturais e mecânicas, a saber: ferritoscopia, difração de raios-x e ultramicrodureza. Os resultados obtidos foram satisfatórios e permitiram estimar o efeito da deformação na transformação martensítica e comparar as técnicas utilizadas.

Palavras-chave: Aços inoxidáveis austenícos; Transformações martensíticas; Martensita induzida por deformação; Análises microestruturais e mecânicas.

\section{MICROSTRUCTURAL AND MECHANICS CHARACTERIZATION FOR THE QUANTIFICATION STRAIN-INDUCED MARTENSITE OF A 304L AUSTENITIC STAINLESS STEEL}

\section{Abstract}

The 304L austenitic steels when processed have their properties affected by martensitic transformations induced by plastic deformation (TRIP effect). The objective of this study was to evaluate this behavior in specimens that have undergone gradual deformation by means of uniaxial tensile tests employing different analysis as ferritoscopy, x-ray diffraction and microhardness. The results obtained by the techniques allowed estimating the effect of the deformation in martensitic transfomation.

Keywords: Austenitic stainless steel; Martensitic transformations; Strain-induced martensite; Mechanical and microstructural analysis.

1 Engenharia Metalúrgica, Mestranda em Engenharia Metalúrgica, Programa de pós-graduação em Engenharia Metalúrgica (PPGEM), Universidade Federal Fluminense, Volta Redonda, RJ, Brasil.

2 Engenharia Química, Doutor em Engenharia Metalúrgica, Professor Adjunto, Departamento de Engenharia Mecânica e PPGEM, Universidade Federal Fluminense, Volta Redonda, RJ, Brasil.

\footnotetext{
* Contribuição técnica ao $69^{\circ}$ Congresso Anual da ABM - Internacional e ao 14ํㅡㄹ ENEMET - Encontro Nacional de Estudantes de Engenharia Metalúrgica, de Materiais e de Minas, 21 a 25 de julho de 2014, São Paulo, SP, Brasil.
} 


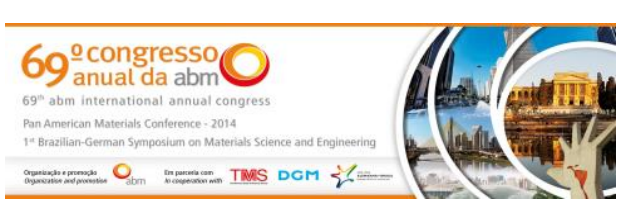

\section{MATERIAIS E MÉTODOS}

O material estudado é um aço inoxidável austenítico 304L processado pela empresa Aperam Inox América do Sul S/A laminado em formas de chapas com a espessura de $1 \mathrm{~mm}$. Esse material é uma liga de $\mathrm{Cr}-\mathrm{Ni}$ com baixo teor de carbono, vide composição química fornecida pela Aperam Inox América do Sul S/A, apresentada na Tabela 1.

Tabela 1. Composição química do aço 304L (Aperam Inox América do Sul S/A)

\begin{tabular}{ccccccccc}
\hline \multicolumn{1}{c}{ ELEMENTOS QUÍMICOS (\% em peso) } \\
\hline $\mathrm{C}$ & $\mathrm{Mn}$ & $\mathrm{Si}$ & $\mathrm{P}$ & $\mathrm{S}$ & $\mathrm{Cr}$ & $\mathrm{Ni}$ & $\mathrm{Mo}$ & $\mathrm{Al}$ \\
\hline 0,018 & 1,27 & 0,479 & 0,0303 & 0,0015 & 18,36 & 8,02 & 0,026 & 0,032 \\
\hline $\mathrm{Cu}$ & $\mathrm{Co}$ & $\mathrm{V}$ & $\mathrm{Nb}$ & $\mathrm{Pb}$ & $\mathrm{B}$ & $\mathrm{Ti}$ & $\mathrm{Sn}$ & $\mathrm{W}$ \\
\hline 0,043 & 0,102 & 0,042 & 0,0071 & 0,001 & 0,006 & 0,0018 & 0,0044 & 0,015 \\
\hline
\end{tabular}

\subsection{Métodos}

Uma vez que o estudo se baseia na quantificação e caracterização da martensita induzida por deformação plástica de um aço inoxidável austenítico 304L, foram realizados ensaios de tração no Laboratório de Ensaios Mecânicos da Escola de Engenharia Industrial Metalúrgica de Volta Redonda (EEIMVR) com auxílio de uma máquina de tração da marca Emic GR 044, com capacidade máxima de $20 \mathrm{KN}$. Os corpos de prova foram ensaiados em uma velocidade $(2,5 \mathrm{~mm} / \mathrm{min})$ e para diferentes deformações a fim de avaliar a fração de martensita transformada em função da deformação. Os ensaios foram interrompidos quando os valores de deformação de engenharia apresentados na Tabela 2 foram alcançados.

Tabela 2. Valores da Velocidade e Deformação

\begin{tabular}{|c|c|c|c|c|c|}
\hline $\begin{array}{c}\text { Velocidade } \\
\text { (mm/min) }\end{array}$ & & $\begin{array}{l}\text { Deformac } \\
\text { Engenha }\end{array}$ & $\begin{array}{l}0 \mathrm{de} \\
(\%\end{array}$ & \multicolumn{2}{|c|}{$\begin{array}{c}\text { Deformação } \\
\text { Verdadeira (\%) }\end{array}$} \\
\hline 2,5 & 20 & 36 & 60 & 80 & 59 \\
\hline
\end{tabular}

A fim de separar apenas a base de medida do comprimento útil dos corpos de prova (cps) $\left(l_{0}=50 \mathrm{~mm}\right)$ após deformação para a caracterização e quantificação da martensita transformada pela deformação plástica, foi utilizado a máquina de corte ISOMET da marca Buehler instalada no Laboratório de Caracterização Microestrutural da EEIMVR na velocidade de 400 rpm com uma carga de $250 \mathrm{~g}$ para evitar a transformação martensítica.

\subsubsection{Técnicas de análise}

Neste tópico são apresentadas as 3 técnicas utilizadas para quantificação da martensita induzida por deformação: Ferritoscopia; Difração de Raios-X e Ultramicrodureza.

\subsubsection{Ferritoscopia}

Após os cortes dos cps, para cada deformação, foi feita medição por indução magnética da fração volumétrica $(\mathrm{V} v)$ de martensita transformada através de um Ferritoscópio tipo Ferritoscope® ${ }^{\circledR}$ FMP30 da marca Fischer, disponível na Universidade Federal Fluminense (UFF/EEIMVR). Para as medições foram feitas

\footnotetext{
* Contribuição técnica ao $69^{\circ}$ Congresso Anual da ABM - Internacional e ao 14ํㅡㄹ ENEMET - Encontro Nacional de Estudantes de Engenharia Metalúrgica, de Materiais e de Minas, 21 a 25 de julho de 2014, São Paulo, SP, Brasil.
} 


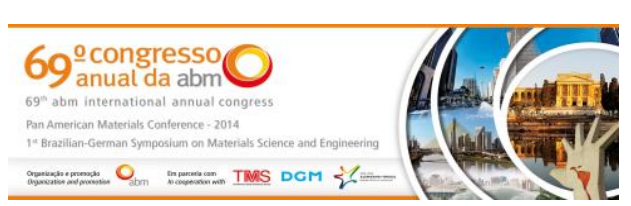

marcações de 5 em $5 \mathrm{~mm}$ no comprimento útil do corpo de prova depois de cortado de acordo com a Figura 2.

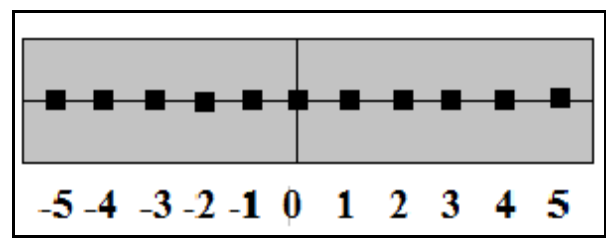

Figura 2. Representação esquemática para medição da fração de martensita transformada com o Ferritoscópio.

\subsubsection{Difração de raios-X}

Foi utilizado o difratômetro do Laboratório de Caracterização Microestrutural da EEIMVR do modelo XRD-6000-SHIMADZU. A radiação incidente foi do tipo Co $\mathrm{K}_{\alpha}$ $(\lambda=1,78897 \AA \hat{A})$ com $30 \mathrm{kV}$ de tensão e $30 \mathrm{~mA}$ de corrente, na Geometria BraggBrentano. A variação das intensidades dos picos serão medidas com base no acoplamento $\theta / 2 \theta$, variando $2 \theta$ de $40^{\circ}$ a $110^{\circ}$ com passo de $0,02^{\circ}$ num modo de varredura contínuo. As fases associadas aos planos serão identificadas como [6]:

- Austenita (CFC - $\gamma$ - A): (111), (200) e (220);

- Martensita ( $\left.\alpha^{\prime}-\mathrm{M}\right)$ : (110), (200) e (211).

A partir dos espectros de DRX, foram calculadas com auxílio da função "Spectroscopy / Baseline and Peaks" disponível no "software OriginPro 8" as medidas relativas à intensidade, área e posição do picos de austenita e martensita a fim de calcular a fração volumétrica de martensita transformada através da intensidade integrada das duas fases.

De acordo com Talonen [7], para calcular essa fração de martensita, foi utilizada a Equação 1:

$$
\frac{\mathrm{I}_{Y}}{\mathrm{I}_{\alpha^{\prime}}}=\frac{\mathrm{R}_{Y}}{\mathrm{R}_{\alpha^{\prime}}} \frac{\mathrm{V}_{Y}}{\mathrm{~V}_{\alpha^{\prime}}}
$$

Onde $I_{Y}$ e $l_{\alpha^{\prime}}$ são as intensidades integradas de austenita e martensita, respectivamente, $R_{\gamma}$ e $R_{\alpha^{\prime}}$ são constantes e $V_{Y}$ e $V_{\alpha^{\prime}}$ são as frações volumétricas das fases austenita e martensita.

Assumindo que $V_{Y}+V_{\alpha^{\prime}}=1$, a fração volumétrica de martensita pode ser encontrada através da Equação 2:

$$
V_{\alpha^{\prime}}=\frac{I_{\alpha^{\prime}} R_{Y}}{I_{Y} R_{\alpha^{\prime}}+I_{\alpha^{\prime}} R_{Y}}
$$

Os valores das constantes $R_{y}$ e $R_{\alpha^{\prime}}$ utilizados para os cálculos são correspondentes ao tipo de radiação incidente utilizada durante o ensaio e também aos picos de maior intensidade. Esses foram calculados por Talonen [7] e estão na Tabela 3.

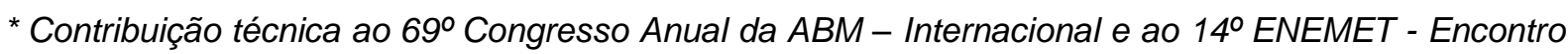
Nacional de Estudantes de Engenharia Metalúrgica, de Materiais e de Minas, 21 a 25 de julho de 2014, São Paulo, SP, Brasil.
} 


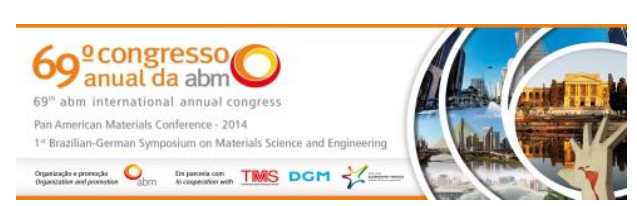

Tabela 3. Valores das constantes $R$ para a austenita( $(\mathrm{Y})$ e martensita $\left(\alpha^{\prime}\right) .[7]$

\begin{tabular}{ccc}
\hline Pico DRX & $R\left(\mathrm{Co} \mathrm{K}_{\alpha^{\prime}}\right)$ & $\mathrm{R}\left(\mathrm{Cr} \mathrm{K}_{\alpha^{\prime}}\right)$ \\
\hline$(220) \mathrm{Y}$ & 36,8 & 47,9 \\
\hline$(110) \alpha^{\prime}$ & 206,2 & \\
\hline
\end{tabular}

\subsubsection{Ultramicrodureza}

Os ensaios de microdureza foram realizados através do UltraMicroDurômetro- DUH 211S da marca Shimadzu instalado no Laboratório Multiusuário de Caracterização de Materiais (LMCM) do Instituto de Ciências Exatas (ICEx) Universidade Federal Fluminense. Foram realizadas medidas com carga de $500 \mathrm{mN}$ A carga aumentava com velocidade tal até atingir $500 \mathrm{mN}$ e permanecia com essa carga durante $25 \mathrm{~s}$ e então era realizado o descarregamento e permanecia ao final durante $25 \mathrm{~s}$.

Para estimar a fração volumétrica da austenita foi usada a Equação 3 [8].

$$
\mathrm{V}_{\mathrm{V}}=1-\frac{\mathrm{V}_{\text {prop }}-\mathrm{V}_{\min }}{\mathrm{V}_{\max }-\mathrm{V}_{\min }}
$$

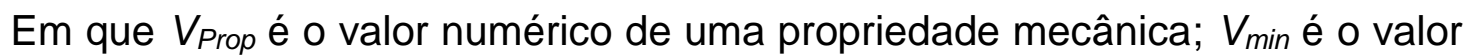
mínimo dessa propriedade mecânica ( no caso aqui seria o valor de dureza com $100 \%$ de austenita); $V_{\max }$ é o valor máximo dessa propriedade mecânica ( no caso aqui seria o valor de dureza com o valor máximo de martensita). Novamente assumindo que $V_{Y}+V_{\alpha^{\prime}}=1$, a fração volumétrica de martensita pode ser encontrada

\section{RESULTADOS E DISCUSSÃO}

A partir dos ensaios de tração uniaxial realizados na velocidade de $2,5 \mathrm{~mm} / \mathrm{min}$ e nas paradas de $20,36,50,60$ e $80 \%$ de deformação de engenharia $(18,31,41,49$ e $59 \%$ de deformação verdadeira) e ruptura. A Figura 3 mostra o comportamento do aço, onde o regime plástico aumenta progressivamente até a ruptura e consequentemente aumentando a fração volumétrica de martensita transformada de acordo com a deformação.

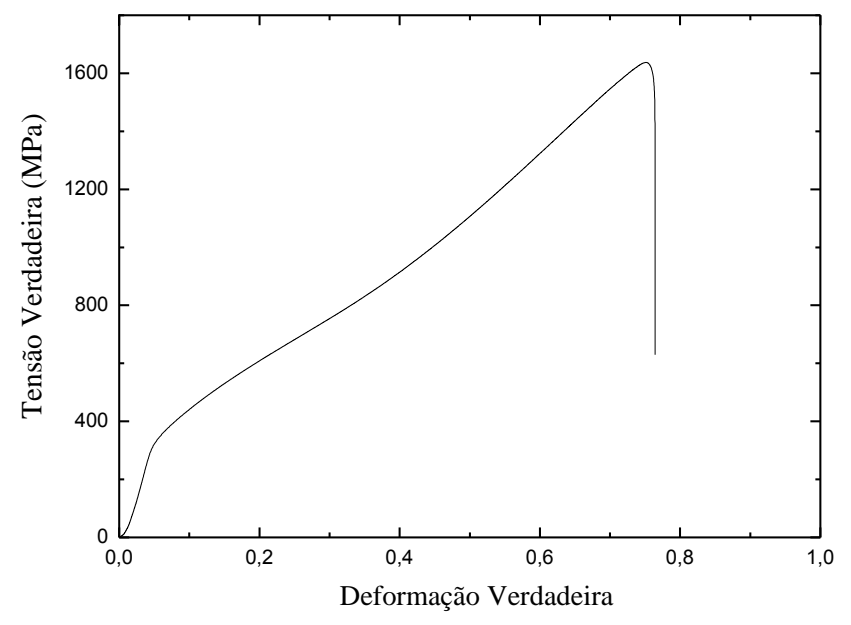

Figura 3. Curva tensão-deformação para ruptura do corpo de prova.

Como era de se esperar a tensão máxima suportada pelo corpo de prova obtida aumenta progressivamente com o aumento da deformação até a ruptura e este comportamento é atribuído ao efeito de transformação de fases induzida por

\footnotetext{
* Contribuição técnica ao $69^{\circ}$ Congresso Anual da ABM - Internacional e ao 14ํㅡㄹ ENEMET - Encontro Nacional de Estudantes de Engenharia Metalúrgica, de Materiais e de Minas, 21 a 25 de julho de 2014, São Paulo, SP, Brasil.
} 
plasticidade, onde parte da fase austenítica se transforma em martensita gerando assim um considerável aumento de resistência mecânica.

\subsection{Ferritoscopia}

A Tabela 4 mostra os valores obtidos de fração volumétrica de martensita ao longo de cada corpo de prova.

Tabela 4. Fração Volumétrica de Martensita para cada deformação

\begin{tabular}{ccccccc}
\hline \multicolumn{6}{c}{ FRAÇÂO VOLUMÉTRICA DE MARTENSITA (\%) } \\
\hline \multicolumn{7}{c}{ Deformação de Engenharia } \\
\hline Pontos & $20 \%$ & $36 \%$ & $50 \%$ & $60 \%$ & $80 \%$ & CR \\
\hline-5 & 1,2 & 5,36 & 12,68 & 20,39 & 30,65 & 0,48 \\
\hline-4 & 1,16 & 5,14 & 12,8 & 20,3 & 30,71 & 0,48 \\
\hline-3 & 1,18 & 4,95 & 12,42 & 20,06 & 30,41 & 0,47 \\
\hline-2 & 1,16 & 4,97 & 12,67 & 20,06 & 30,4 & 0,47 \\
\hline-1 & 1,15 & 5,01 & 12,45 & 19,98 & 30,01 & 0,48 \\
\hline 0 & 1,16 & 5,02 & 12,12 & 19,58 & 29,91 & 0,49 \\
\hline 1 & 1,18 & 5,03 & 12,31 & 19,73 & 30,01 & 0,48 \\
\hline 2 & 1,16 & 5,01 & 12,12 & 19,88 & 29,98 & 0,48 \\
\hline 3 & 1,16 & 5,00 & 12,73 & 20,41 & 30,37 & 0,48 \\
\hline 4 & 1,17 & 5,11 & 13,01 & 20,32 & 30,4 & 0,47 \\
\hline 5 & 1,18 & 5,30 & 12,89 & 20,49 & 29,98 & 0,48 \\
\hline Média & 1,17 & 5,09 & 12,56 & 20,11 & 30,26 & 0,48 \\
\hline
\end{tabular}

Percebe-se que os valores para $V_{v}$ de martensita aumentam de acordo com 0 aumento da deformação plástica durante o ensaio de tração. Cardoso [9] e Hecker [4] também encontraram o mesmo comportamento para essa curva que relaciona a fração volumétrica de martensita transformada em função da deformação. A Figura 4 ilustra melhor essa evolução utilizando os valores médios obtidos na Tabela 4.

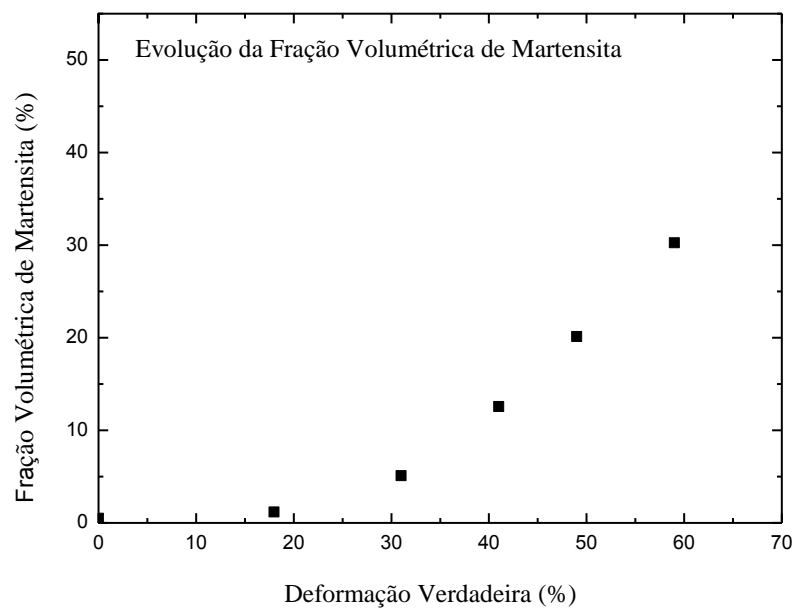

Figura 4. Evolução da fração volumétrica de martensita com a deformação verdadeira.

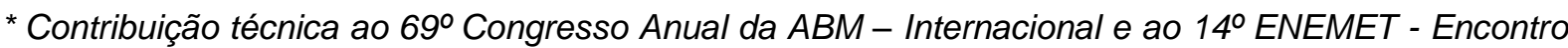
Nacional de Estudantes de Engenharia Metalúrgica, de Materiais e de Minas, 21 a 25 de julho de 2014, São Paulo, SP, Brasil.
} 


\subsection{Difração de Raios-X}

Os ensaios de difração de raios-x tiveram como objetivo identificar as fases presentes nas condições de estudo e verificar a evolução da transformação de austenita para martensita através dos picos de difração de cada uma das fases associadas às orientações e planos dos grãos paralelos a superfície da chapa que são consumidas (austenita) e formadas (martensita).

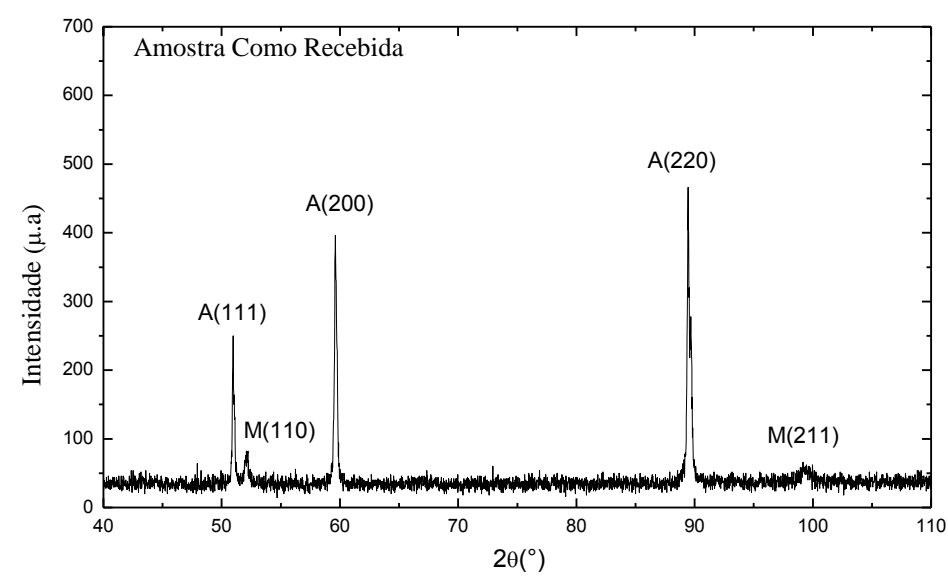

Figura 5. Espectro de difração de raios-x da amostra como recebida.

A Figura 5 apresenta o difratograma para amostra como recebida. Verifica-se que existem três picos predominantes associados a austenita relativos aos planos difratados, que são: (111), (200) e (220) e são representados pela letra A. E para a martensita, os picos são menos intensos e associados aos planos (110) e (211) e representados pela letra $M$. Esse fato confirma a pequena porcentagem de martensita, aproximadamente $0,48 \%$, detectada pelo Ferristoscópio antes do ensaio de tração. A amostra apresenta quase $100 \%$ de austenita, uma vez que as intensidades dos seus planos são maiores que as da martensita, que é bem inferior. A Figura 6 apresenta os difratogramas para os corpos de prova ensaiados com as paradas de 20, 36, 50, 64 e $80 \%$ de deformação de engenharia. Observa-se que com o aumento gradativo da deformação plástica, surge um novo plano para a martensita, que é o plano (200) e também que a intensidade dos picos de austenita vai diminuindo enquanto que os de martensita vão aumentando progressivamente. Isso acontece porque com a deformação a fração $V_{V}$ de martensita transformada também aumenta.

\footnotetext{
* Contribuição técnica ao $69^{\circ}$ Congresso Anual da ABM - Internacional e ao 14ํㅡㄹ ENEMET - Encontro Nacional de Estudantes de Engenharia Metalúrgica, de Materiais e de Minas, 21 a 25 de julho de 2014, São Paulo, SP, Brasil.
} 

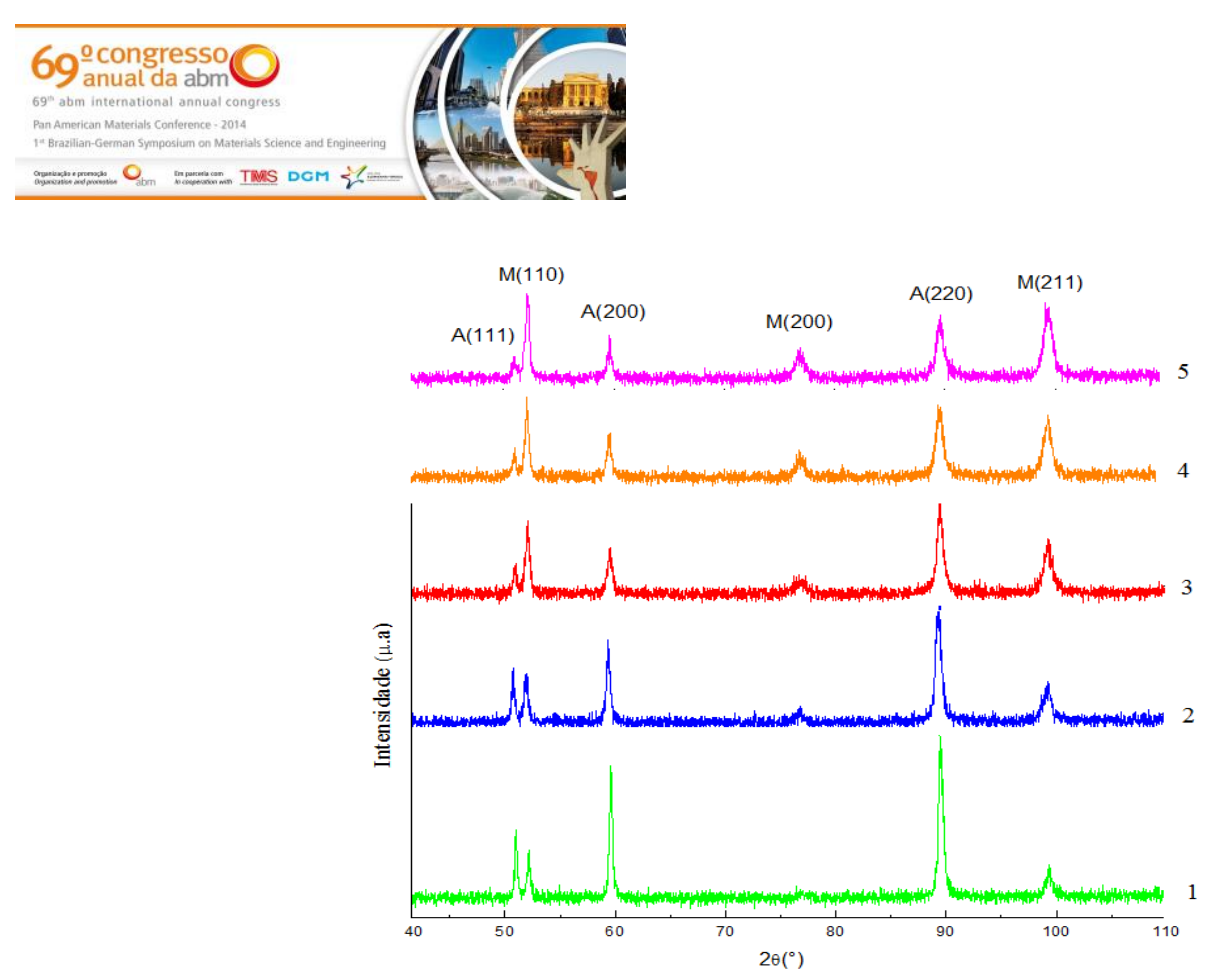

Figura 6. Espectro de difração de raios-x para 1: $20 \%, 2: 30 \%, 3: 50 \%, 4: 64 \%$ e $5: 80 \%$ de deformação de engenharia.

Com os resultados dos difratogramas, foi estimado o valor da fração volumétrica de martensita transformada em cada deformação, através da Equação 2. Os valores de $R_{\alpha^{\prime}}=206,2$ e $R_{y}=36,8$ utilizados da Tabela 3 foram os que correspondem aos picos de maior intensidade para a austenita (220) e martensita (110).

Utilizando a função "Spectroscopy / Baseline and Peaks" disponível no "software OriginPro 8" obteve-se os valores das áreas integradas para os respectivos picos dos planos de maior intensidade para de austenita e martensita. Esses valores estão na Tabela 5, onde também estão os resultados obtidos para fração volumétrica de martensita, utilizando a Equação 2.

Tabela 5. Valores de $\mathrm{V}_{v}$ de martensita de acordo a intensidade da área integrada para os planos de maior intensidade.

\begin{tabular}{|c|c|c|c|}
\hline Amostra & Pico DRX & $\begin{array}{c}\text { Intensidade da Área } \\
\text { Integrada }\end{array}$ & $\begin{array}{c}\text { Fração Volumétrica de } \\
\text { Martensita (\%) }\end{array}$ \\
\hline \multirow{2}{*}{$\begin{array}{c}\text { Como } \\
\text { Recebida }\end{array}$} & $(110) \alpha^{\prime}$ & $\alpha^{\prime}=2,28$ & \multirow{2}{*}{0,48} \\
\hline & $(220) \mathrm{y}$ & $\mathrm{I} y=82,09$ & \\
\hline \multirow{2}{*}{1} & $(110) \alpha^{\prime}$ & I $\alpha^{\prime}=24,32$ & \multirow{2}{*}{1,75} \\
\hline & $(220) \mathrm{y}$ & $\mathrm{I} \gamma=144,52$ & \\
\hline \multirow{2}{*}{2} & $(110) \alpha^{\prime}$ & I $\alpha^{\prime}=21,48$ & \multirow{2}{*}{5,04} \\
\hline & $(220) \mathrm{y}$ & I $y=72,28$ & \\
\hline \multirow{2}{*}{3} & $(110) \alpha^{\prime}$ & $\alpha^{\prime}=58,22$ & \multirow{2}{*}{11,48} \\
\hline & $(220) y$ & $\mathrm{I} \gamma=80,09$ & \\
\hline \multirow{2}{*}{4} & $(110) \alpha^{\prime}$ & $\alpha^{\prime}=48,46$ & \multirow{2}{*}{21,64} \\
\hline & $(220) \mathrm{V}$ & $I y=31,31$ & \\
\hline \multirow{2}{*}{5} & $(110) \alpha^{\prime}$ & I $\alpha^{\prime}=84,67$ & \multirow{2}{*}{30,94} \\
\hline & $(220) y$ & $\mathrm{I} y=33,72$ & \\
\hline
\end{tabular}

Com os resultados obtidos pode-se concluir que os valores estão de acordo com os obtidos no Ferritoscópio. Para melhor representação, a Figura 7 mostra o gráfico da comparação entre as duas técnicas utilizadas. Através de um ajuste linear é possível obter o coeficiente de determinação, $R^{2}$. $O R^{2}$ varia entre 0 e 1 , indicando, em percentagem, o quanto o modelo consegue explicar os valores observados. Quanto

\footnotetext{
* Contribuição técnica ao 69ำ Congresso Anual da ABM - Internacional e ao 14ํㅡㄹ ENEMET - Encontro Nacional de Estudantes de Engenharia Metalúrgica, de Materiais e de Minas, 21 a 25 de julho de 2014, São Paulo, SP, Brasil.
} 


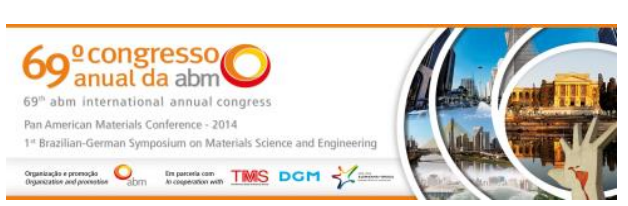

maior o $\mathrm{R}^{2}$, mais explicativo é modelo, melhor ele se ajusta à amostra. Para a curva do gráfico da Figura 7 o valor de $R^{2}$ foi de 0,994. Indicando uma excelente correlação entre as duas técnicas utilizadas.

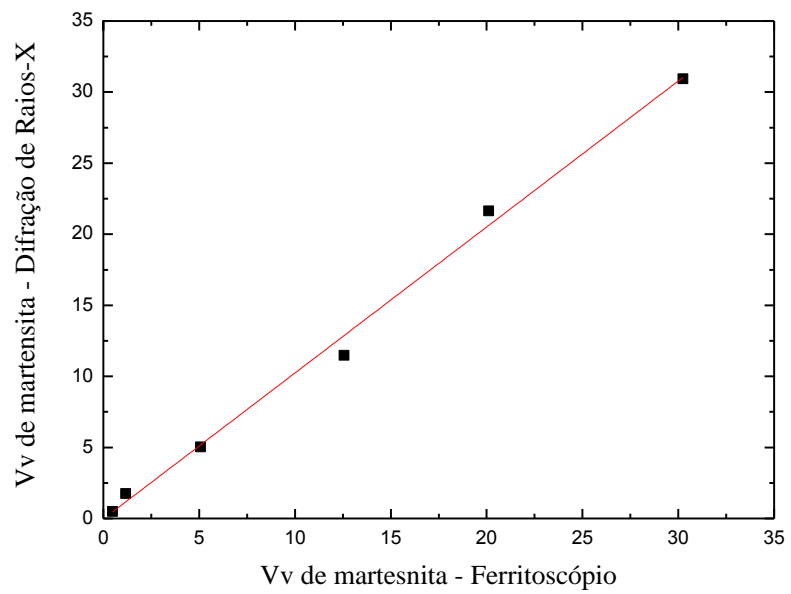

Figura 7. Comparação das medidas de fração volumétrica de martensita obidas via: Difração de Raios-X e Ferritoscópio.

$A$ equação obtida que relaciona $V_{V}$ de martensita obtida através de DRX com a obtida via ferritoscopia é:

$$
V_{V(\text { DRX })}=-0,034+1,027\left(V_{V_{\text {Ferritoscópio }}}\right)
$$

\subsection{Ultramicrodureza}

A Figura 8 mostra o gráfico correspondente a ensaios de ultramicrodureza realizados para cada amostra que sofreu ensaio de tração.

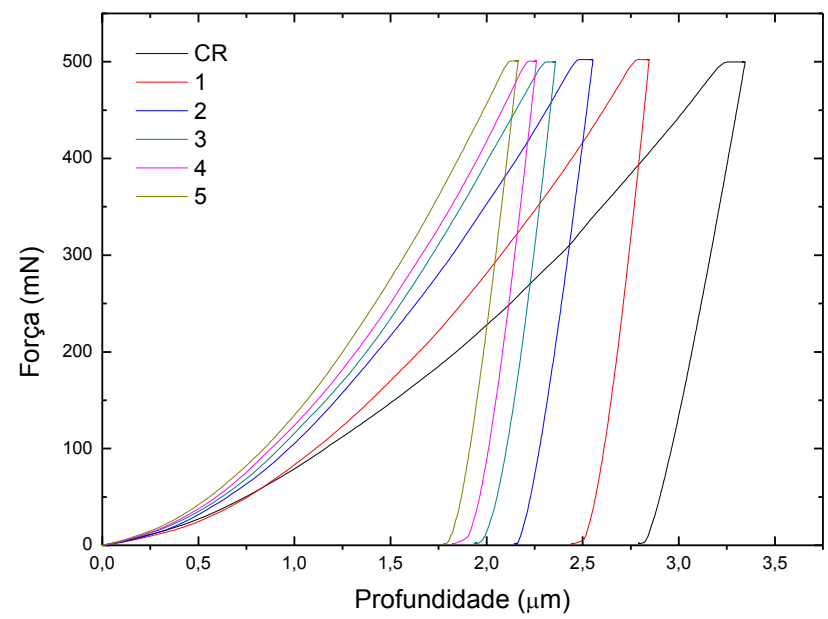

Figura 8: Curvas de indentação de dureza para o material como recebido e amostras deformadas para 1: $20 \%, 2: 30 \%, 3: 50 \%, 4: 64 \%$ e 5: $80 \%$ de deformação de engenharia.

A Figura 8 mostra as curvas de indentação para a amostra como recebida e amostras deformadas. A curva de indentação, também designada como curva carga-descarga representa a evolução da carga aplicada com a profundidade de indentação durante o ensaio de dureza. É constituída por uma fase de carga seguida

* Contribuição técnica ao 69 Congresso Anual da ABM - Internacional e ao 14ํㅡㄹ ENEMET - Encontro Nacional de Estudantes de Engenharia Metalúrgica, de Materiais e de Minas, 21 a 25 de julho de 2014, São Paulo, SP, Brasil. 


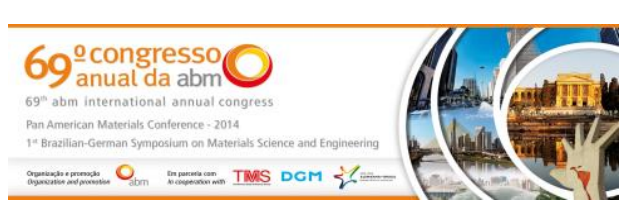

experimental que $30,26 \%$ e o segundo maior valor calculado se relaciona com um valor $X$ e assim sucessivamente mantendo sempre os valores 100 e 30,26 e variando os outros valores.

Os valores obtidos pela técnica de dureza não estão coerentes com os obtidos no Ferritoscópio. A Figura 10 mostra o gráfico da comparação entre as duas técnicas utilizadas. $O$ coeficiente de determinação, $R^{2}$ foi de 0,68 . Indicando que não existe uma boa correlação entre as duas técnicas utilizadas.

Tabela 6. Valores de fração volumétrica de martensita

\begin{tabular}{ccc}
\hline Amostras & $\begin{array}{c}\mathrm{V}_{\mathrm{V}} \text { de Martensita }(\%) \text { pela } \\
\text { Fórmula }\end{array}$ & $\begin{array}{c}\mathrm{V}_{\mathrm{V}} \text { de Martensita }(\%) \\
\text { corrigida }\end{array}$ \\
\hline Como Recebida & 0 & 0 \\
\hline 1 & 32 & 9,8 \\
\hline 2 & 68 & 20,8 \\
\hline 3 & 71 & 22,1 \\
\hline 4 & 89 & 27,6 \\
\hline 5 & 100 & 30,94 \\
\hline
\end{tabular}

O $V_{v}$ obtido via dureza superestima inicialmente o valor de $V_{v}$ obtido via ferrritoscópio e DRX, Figura 7. Duas questões precisam ser levadas em conta ao encontrar $V_{v}$ de martensita via dureza. Primeiramente neste trabalho não se obteve $100 \%$ de martensita em nenhuma amostra. O maior valor de martensita foi em torno de $30 \%$, o que foi um obstáculo para usar diretamente a Equação 3. Em segundo lugar foi proposto uma regra de três, tendo a hipótese que a transformação martensítica teria um comportamento linear, o que não se mostrou verdadeiro.

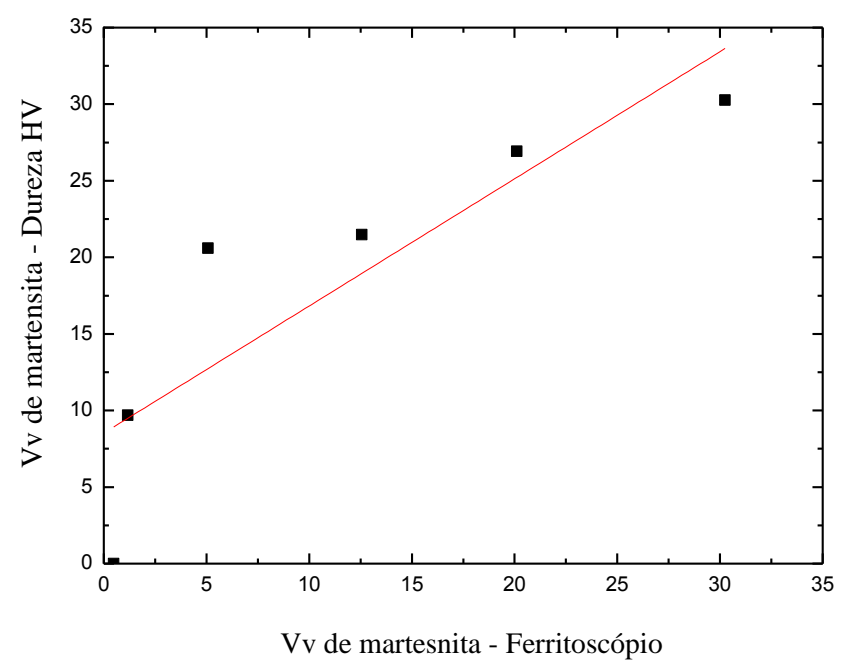

Figura 10. Comparação das medidas de fração volumétrica de martensita obidas via: Dureza e Ferritoscópio.

\section{CONCLUSÃo}

O estudo realizado permitiu criar uma metodologia que quantificasse a fração volumétrica $\left(V_{v}\right)$ de martensita induzida por deformação. As técnicas de Ferritoscopia e Difração de Raios-X foram satisfatórias, uma vez que se obtiveram valores de $\mathrm{V}_{\mathrm{V}}$ de martensita coerentes e bem próximos em ambas as técnicas. Ou seja, através do ferritoscópio é possível estimar $V_{V}$ de martensita. Os ensaios de Ultramicrodureza demonstraram que existe uma correlação, onde quanto maior $V_{V}$

* Contribuição técnica ao $69^{\circ}$ Congresso Anual da ABM - Internacional e ao 14ํㅡㄹ ENEMET - Encontro Nacional de Estudantes de Engenharia Metalúrgica, de Materiais e de Minas, 21 a 25 de julho de 2014, São Paulo, SP, Brasil. 


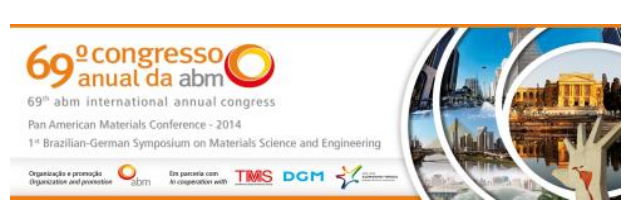

transformada maior a dureza. Entretanto, os valores de $\mathrm{V}_{\mathrm{V}}$ de martensita obtidos a partir da dureza foram maiores que os valores obtidos através das outras técnicas utilizadas. Para o uso desta técnica seria necessário obter um número maior de amostras e principalmente com maiores frações volumétricas de martensita até $100 \%$, para que pudesse ocorrer a validação ou não da técnica.

\section{Agradecimentos}

Os autores agradecem ao CNPQ pelo apoio financeiro no âmbito do projeto de pesquisa do edital Universal 014/2010, a empresa Aperam Inox América do Sul S/A, Instituto de Ciências Exatas (ICEx) Universidade Federal Fluminense. Capes pela bolsa de mestrado concedida a autora.

\section{REFERÊNCIAS}

1 Gallée S, Pilvin P. Deep drawing simulation of a metastable austenitic stainless steel using a two-phase model. Journal of Materials Processing Technolog. 2010; 210: 835843.

2 Smith WF. Principles of Materials Science and Engineering. 3. ${ }^{a}$ edição.New York: McGraw-Hill; 1996.

3 Dan WJ, Zhang WG, Li SH, Lin ZQ. A model for strain-induced martensitic transformation of TRIP steel with strain rate. 2007; 40: 101-107.

4 Hecker SS, Stout MG, Staudhammer KP, Smith JL. Effects of strain state and strain rate on deformation-induced transformation in 304 stainless steel.Part I. Magnetic measurements and mechanical behavior. Metall. Trans,1982. A13: 619-626.

5 Tavares SSM, Pardal JM, Silva MJG, Abreu, HFG, SILVA MR. Deformation induced martensitic transformation in a 201 modified austenitic stainless steel. Materiala Characterization .2009; 60: 907-911.

6 Paula AS, Cardoso MC, Vieira TF, Andrade JG, Monteiro GA, Moreira LP, Freitas MC. Kinects and Structural Evolution of 304l Stainless Steel with Trip Effect When Submitted to Uniaxial Tensile Test Under Distinct Strain Rates. ABM International Congress. 2012; 67: 2050-2061.

7 Talonen J, Aspegren $\mathrm{P}$, Hännien $\mathrm{H}$. Comparison of different methods for measuring strain induced $\alpha^{\prime}$ martensite content it austenitc steels. Materials science and Technology. 2004; 20: 1506-1512.

8 Ferreira RAS. Transformação de Fase: aspectos científicos e morfológicos. Ed. Universitária da UFPE, 2002.

9 Cardoso MC. Avaliação do Comportamento Plástico de uma Chapa de Aço Inoxidável Austenítico 304L por meio de Ensaios de Tração Uniaxial e Curva Limite de Conformação (Dissertação de Mestrado). Rio de Janeiro: Escola de Engenharia Industrial de Volta Redonda, 2012.

\footnotetext{
* Contribuição técnica ao $69^{\circ}$ Congresso Anual da ABM - Internacional e ao 14ํㅡㄹ ENEMET - Encontro Nacional de Estudantes de Engenharia Metalúrgica, de Materiais e de Minas, 21 a 25 de julho de 2014, São Paulo, SP, Brasil.
} 